\title{
DEFENSE METEOROLOGICAL SATELLITE PROGRAM
}

\author{
Colonel Justin A. Curtis, Deputy for Defense Meteorological Satellite System \\ Colonel Louis J. Adams, Assistant Deputy for Defense Meteorological Satellite System \\ Headquarters USAF Space Division \\ Los Angeles, CA 90009-2960, USA
}

\begin{abstract}
The DMSP is a total satellite system composed of spacecraft with meteorological sensors, an earthbased command and control network, fixed and mobile user stations, and a communication network linking the various segments together.
\end{abstract}

\section{INTRODUCTION}

The mission of DMSP is to provide global meteorological data to Tri-Service Commanders in support of worldwide military operations, both strategic and tactical, and to advance spaceborne meteorological sensing technology to meet changing Department of Defense requirements. The Space Segment consists of two satellites in 830 kilometer sun synchronous polar orbits each carrying a payload of various meteorological sensors. The Satellite Operations Center is located at Offutt AFB, Nebraska, with earth terminals located at Loring AFB, Maine and Fairchild AFB, Washington. Real-time cloud imagery data is transmitted directly from the spacecraft to Air Force, Navy, and Marine Corps ground tactical terminals and Navy carriers located throughout the world. Stored or playback meteorological data is transmitted to centralized facilities at the Air Force Global Weather Central (AFGWC) at Offutt AFB, Nebraska, and the Navy Fleet Numerical Oceanography Center (FNOC) in Monterey, California. At these centralized facilities, global cloud cover and other meteorological sensor data can be merged with more conventional data and distributed to provide weather support throughout the Department of Defense. In support of the command and control, telemetry and meteorological data, the system uses domestic communication satellites and land lines to interconnect the various segments in a cohesive and responsive manner.

\section{SPACE SEGMENT}

The spacecraft consists of a three-axis zero momentum vehicle with an on-orbit weight of 775 kilograms and 3.5 meters in length exclusive of solar panels. It has a pointing accuracy and knowledge of $0.01^{\circ}$ and a 30-month mean mission duration.

\section{Sensor}

The Operational Linescan System (OLS) is the primary meteorological sensor. The OLS is a complete self-contained data collection system consisting of an oscillating scanning radiometer and a data processing and storage subsystem which provide meteorological imagery data (cloud cover) on a global basis. The system collects day and night earth scene image data which is then transmitted in real time and/or stored over multi-orbits.

Earth scene data is sensed by the OLS in two complementary spectral bands: visible light and thermal infrared. In each channel the scene resolution across scan is made nearly constant by using a variable instantaneous field of view in conjunction with a sinusoidally varying scan motion. For global coverage the nominal "smoothed mode" resolution is $2.78 \mathrm{~km}$ in each channel and for selected regional coverage with higher resolution a nominal $0.56 \mathrm{~km}$ "fine mode" is provided in each channel.

The visible channel senses scene radiance in the 0.4 to 1.1 micrometer spectral band for scene illuminations ranging from sub-solar to sub-lunar at quarter moon, a range of over ten million to one. The infrared channel senses scene radiance in the 10 to 13 micrometer spectral band corresponding to a scene temperature range from $190^{\circ} \mathrm{K}$ to $310^{\circ} \mathrm{K}$. The output data is made linear with temperature over the dynamic range.

The visible channel detectors are three-segment silicon photoconductive PIN diodes for daytime scenes and a cesiated gallium arsenide opaque photocathode for nighttime scenes. The infrared detector is a two-segment MercuryCadmium-Telluride photoconductive detector passively 
cooled to a temperature near $108^{\circ} \mathrm{K}$ and maintained within $\pm 0.1^{\circ} \mathrm{K}$ of the chosen set point. The imagery data across the scan path is collected in the form of discrete picture elements (pixels) and converted into one of either 64 or 256 grey shades.

The program and data storage are provided by the OLS memory consisting of $3 \mathrm{~K}$ of ROM and 13K of CMOS RAM. In addition, there is $16 \mathrm{~K}$ of core RAM memory. Processed meteorological data is stored on four magnetic tape recorders, each having a storage capacity of $1.67 \times 10^{9}$ bits.

In addition to the OLS, the spacecraft has other meteorological sensors, some of which may employ advanced spaceborne meteorological sensing technology. These include atmospheric sensors to provide vertical temperature and moisture profiles and to measure ocean surface wind, land surface moisture, integrated water vapour content, rain and cloud water content. Additional space and ionospheric sensors detect thermal ion and electron irregularities, auroral producing electron fluxes; provide the location and energy spectrum of gamma and $x$-ray sources in the atmosphere and measure the earth's radiation and direction of the earth's magnetic field.

\section{Communications and Telemetry}

For meteorological data transmission the spacecraft communications subsystem incorporates three independent S-band links using PCM/PSK transmission at $1.024 \mathrm{Mbps}$ real time and $2.66 \mathrm{Mbps}$ playback data rates. The transmitters are 5 -watt solid state units used in conjunction with crosseddipole directional antennas mounted on the earth-facing side of the spacecraft. In addition, there are redundant S-band telemetry links for spacecraft status, one using a high gain directional antenna while the other is omnidirectional with two half-turn, half-wave helical antennas. This telemetry link uses PCM/PSK/PM modulation.

The uplink command system operates at a $2 \mathrm{~K}$ rate and has ternary MFSK/AM modulation. Its main component is the Receiver Demodulator Unit (RDU) that receives commands, decodes them, checks their validity, and routes them to the rest of the satellite. The receive L-band antennas are similar to the telemetry omnidirectional units.

The telemetry unit is a solid-state CMOS system under microprocessor control using 4K of PROM and 2K of RAM. With three commandable data rates of 2,10 , or $60 \mathrm{Kbps}$, it also has numerous commandable submodes, i.e., ability to obtain OLS, spacecraft and telemetry system computer memory dumps, to sample analog channels using higher sampling rates, and to rearrange analog and discrete channel assignments.

\section{Command and Control}

The command and control subsystem is the focal point for all spacecraft functions and operations. The subsystem also includes computers and memories which control all spacecraft functions, an input/output unit (controls interface unit) that passes/receives all information to/from the computers, a stable spacecraft clock, and processing to decode and distribute all commands. Two central processing units are used, each currently having $28 \mathrm{~K}$ read/write memory with single bit error detection. This increases to $64 \mathrm{~K}$ of memory with two bit error detection, single bit correction in later spacecraft. The subsystem multiplexes its own internal telemetry data stream, composed of attitude and control parameters for insertion into the telemetry data format.

The software elements of the command and control subsystem process command messages and data loads, maintain the status of the hardware, and generate control signals to all elements of the spacecraft, both hardware and software. The Ascent Guidance Software provides the navigation, upper stage thruster control, velocity trim and final orientation maneuvers during ascent through orbital insertion. The Orbit Mode Software provides attitude determination and control after achieving orbit.

\section{Attitude Determination and Control}

The accurate ( 0.01 degree) three-axis attitude determination and control subsystem provides precise pointing of the sensor payload. Three on-board orthogonal gyroscopes (with a fourth skewed unit for backup) measure short-term changes in spacecraft rotation rate; a star sensor measures long-term drift. An on-board processor stores ephemeris data and computes the spacecraft attitude. To enhance pointing accuracy, extensive star catalogs and ephemeris tables are periodically transmitted to the spacecraft from the ground. Attitude control is provided by three reaction wheels in an active closedloop configuration (with a fourth for back-up), magnetic coils for unloading excess momentum, and $\mathrm{GN}_{2}$ thrusters for transient momentum disturbances. In the event of failure of the Inertial Measurement Unit or Celestial Sensor Assembly, a lower-accuracy (0.1 degree) three-axis attitude determination and control is available. It is provided by the Earth Sensor Assembly and the Sun Sensor Assembly.

\section{Other Spacecraft Subsystems}

The other subsystems consist of a power system which includes photovoltaic solar cells, two nickel-cadmium batteries and power conditioning electronics, a single-axis control system to allow the solar array to point to the sun, and both passive and active thermal control systems.

\section{GROUND COMMAND AND CONTROL SEGMENT}

The DMSP spacecraft has an extensive worldwide system available to provide support for meeting the mission objectives. In addition to the three earth terminals and domestic communication satellite network mentioned earlier, it has the added resources of the Air Force Satellite Control Facility's worldwide Remote Tracking Stations and the extensive DOD communication satellite network to provide telemetry and command backup. All of these resources are presently linked via terrestrial or satellite links.

\section{Satellite Operations Center}

The Satellite Operations Center (SOC) at Omaha is the primary command and control site and contains the personnel and systems necessary to conduct all mission planning, spacecraft commanding, telemetry ingest and post-pass analysis. 
Mission planning encompasses all tasks associated with data generation and scheduling for the $\mathrm{C}^{3}$ ground system and satellite operating activities. It synthesizes the user's data needs, engineering requests for special satellites tests, and processes satellite status information to create the operational data files necessary to control the DMSP satellite system. These requirements and operational constraints are then incorporated into the automated Mission Planning Data Generation and Verification System (PLANS).

The Stored Telemetry Processing System (STPS) provides data analysts with the means to conduct post-pass, long-term telemetry analysis of spacecraft anomalies. The system operates either interactively or in the batch mode. For realtime telemetry, the SOC utilizes specially built decommutators to separate spacecraft telemetry data for computer processing. Real-time processing provides conversion to engineering units and identification of out-of-limit parameters, all displayed on color graphic terminals. Once the satellite contact period is over, the stored telemetry and the real-time telemetry are merged into the revolution-by revolution telemetry data base, and are the accessible for post-pass computer analyses.

\section{Satellite Communications}

At each of the three earth terminals, the meteorological and telemetry data are multiplexed together with site status data and a digital voice signal into a single 3.072 Mbps data stream. The 3.072 Mbps data stream from the Hawaii Tracking Station does not contain digital voice or site status. The data stream from the three sites are uplinked to the Westar satellite, leased from Western Union by the American Satellite Corporation, and relayed to terminals located at AFGWC and FNOC. Both sites receive all the data contained on each data stream. The command channel from the Omaha Satellite Operations Center to the two Command Readout Stations (CRSs) is a single time division multiplexed (TDM) channel that contains a composite of command, control and digital voice at $230.4 \mathrm{Kbps}$. The command data stream is converted from a serial binary to serial ternary form at each CRS and uplinked to the spacecraft.

The primary links to the CRSs are backed up by terrestrial land lines that are capable of handling the same types of command and telemetry data as Westar but at a lower data rate. The terrestrial lines do not provide mission data to AFGWC or FNOC. In addition, there are command/telemetry back-up links to/from the Air Force Satellite Control Facility, the spacecraft factory and Vandenberg AFB for launch and test support.

\section{Command Readout Stations}

The Command Readout Stations, located at Loring AFB, Maine and Fairchild AFB, Washington, consist of earth terminals for accessing and receiving data from the spacecraft. The antenna system has a 12 -meter reflector with a gain/temperature of $20.75 \mathrm{db} /{ }^{0} \mathrm{~K}$ and uses pseudo-monopulse autotracking.

In addition to processing and transmitting commands in real time, the terminal at Fairchild AFB has a local commanding capability by means of command data shipped in advance from the SOC PLANS computer. The extent of this stand-alone operation is dependent upon the quantity of command data resident at the local site.

The downlink function receives, stores and forwards all incoming S-band telemetry and mission data signals to the two centralized user segments.

\section{USER SEGMENTS}

\section{Air Force Global Weather Central}

The Air Force Global Weather Central (AFGWC) is the largest military meteorological center in the world and provides worldwide meteorological and space environmental support to not only the Air Force but to the Army and a variety of Defense Department agencies as well. In addition, AFGWC backs up the National Weather Service's National Meteorological Center and the National Severe Storms Forecast Center.

Over 140,000 weather reports per day are gathered from conventional meteorological sources (surface observations, rawinsondes, radar and aircraft) and are relayed via the high-speed Automated Weather Network (AWN). These data are blended with information available from military and civilian meteorological satellites to construct an integrated environmental data base in real time. A series of computer programs are employed to construct models of the existing atmosphere and ionosphere and to project changes that will occur in the future. These basic meteorological and space environmental tools are made available to scientists and technicians for application to specific problems encountered by operations in the aerospace environment.

AFGWC is not an automated production center; rather, it is a computer-based operation heavily reliant upon the interaction between people and computers to produce accurate and complete services designed for operational support requirements. Products and services provided by AFGWC include: meteorological advice; aviation, terminal and target forecasts; prediction of severe weather; automated flight planning; exercises and special mission support; computations for ballistic missile systems; and analyses and predictions of solar-geophysical events which affect systems operating any place in or through the near-earth all the way into deep-space environments.

All these data sources feed into AFGWC which, operating under a build and apply philosophy, devotes much of its resources to building a variety of data bases. The remaining resources go toward meteorological applications of these various data bases. Among the many thousands of products created and transmitted from AFGWC daily are MAC computer flight plans, other computer flight plans, terminal forecasts, facsimile charts, some 100 to 150 point weather warnings, and as many as 150 requests for special forecast assistance.

DMSP satellite data is used in a variety of ways at AFGWC to support both meteorological and space environmental customers. Global cloud imagery, visual and infrared, is available in several different forms. The forecaster may receive a positive transparency for use on a light table or a hard copy print on which analyses can be done or the image may be displayed on the Satellite Data 
Handling System (SDHS). The imagery is also processed directly into the Satellite Global Data Base (SGDB). With each new batch of stored data into AFGWC, this data base is updated. The meteorologists then use the SGDB to reconstruct computer-generated images of any area of the globe or merge this data with conventional data to produce the world's only three-dimensional cloud analysis model. With additional input from numerical forecast models, a short-term cloud forecast can be generated. The recent completion of the Satellite Data Handling System (SDHS) at the AFGWC will dramatically improve the ability of forecasters to interact with satellite and conventional weather data to produce the products mentioned. This highly interactive state-of-the-art network of 35 work stations, each with four CRTs driven by 38 mini and midi computers, will replace much of the manual effort hard copy currently required at AFGWC, saving 108 manpower slots and speeding up the overall production cycle. AFGWC is in the process of transitioning its production tasks to the SDHS, and should be fully transitioned by late 1987 .

The tropical meteorologist at AFGWC has found DMSP cloud imagery to be particularly useful. In areas of the world where there are virtually no conventional observations, typhoon location and tracking have been traditionally accomplished by aircraft reconnaisance. However, as this resource has decreased, DMSP has become increasingly more important to both naval and fixed base forces.

DMSP data has also been found very useful in the construction of hemispheric weather depiction charts. These charts show areas of cloudiness, cloud type, icing, and turbulence. Wind direction vectors can also be extracted from DMSP pictures and used for upper-air analyses performed at AFGWC. The severe weather forecaster also uses cloud imagery to locate areas of potential instability and to assist in the preparation of point weather warnings.

In addition to cloud imagery, the forecaster has additional DMSP data available from a variety of infrared and microwave sounders and imagers. Among the types of information these sensors provide are vertical temperature profiles. By sensing infrared and microwave radiation transmitted by the earth and by atmospheric gases $\left(\mathrm{CO}_{2}, \mathrm{H}_{2} \mathrm{O}, \mathrm{O}_{2}\right)$, the meteorologist can reconstruct temperature profiles. This type of information has long been used as the basis for atmospheric analysis and forecasting. Prior to the advent of satellite remote sensing, temperature profiles were obtained solely from instrumented balloons, with severely limited coverage. With DMSP we now have worldwide coverage which fills in the data void regions. By improving our initial analyses we also improve our numerical forecasts.

\section{Navy Fleet Numerical Oceanography Center}

The Navy Fleet Numerical Oceanography Center (FNOC) in Monterey, California, receives the identical mission data as the Air Force Global Weather Central via AMSAT relay. The environmental data can then be transmitted via terrestrial links to Oceanography Command Centers at Pearl Harbor, Hawaii; Norfolk, Virginia; Suitland, Maryland; Guam and Spain, for detailed processing for use in regional forecasting support. The DMSP satellite data is also used in a variety of ways at FNOC, both alone and merged with more conventional data to provide meteorological support to Navy ships and facilities worldwide.

\section{Mobile Terminals}

The Mark IV is the latest generation, mobile, receive-only, ground terminal to provide real-time DMSP visual and infrared cloud imagery data to theater decision makers. It is a compact unit consisting of a 3-meter parabolic antenna, 6-meter van, and an auxiliary power generator, designed to be transported aboard C-130 or C-141 aircraft. It has automated acquisition and tracking and a low noise front end with a gain/temperature of $11.3 \mathrm{db} /{ }^{0} \mathrm{~K}$. The Mark IV has the capability to track and process data from both the DMSP and NOAA weather satellites. Hard copy data can then be provided to at least four remote locations over cable and telephone circuits. Mark IVs are intended to be co-located with theater commanders and are planned for deployment to Europe, Alaska, Pacific, Far East, Central America, United Kingdom, and CONUS.

The 1.024 Mbps serial, real-time data is demodulated and formatted for processing while being simultaneously recorded along with time code information on an analog tape recorder. The formatting consists of a serial data synchronization process, a serial-to-parallel data conversion, and a decommutation process. The decommutation process extracts the visual or infrared imagery data and the other auxiliary data. The imagery data is the primary data input for display generation, with the auxiliary data being used for appropriate image correction. The visual or infrared images can be enlarged, enhanced, labeled and gridded within 30 seconds of a satellite pass.

In the real-time mode, the imagery data is formatted, corrected for earth curvature and wow/flutter effects, enhanced and overlayed, and sent to hard and soft copy displays. The hard copy is a laser scanned, dry-processed film transparency suitable for light table viewing and photographic reproduction, while the soft copy is a CRT monitor display.

The capability to search through the entire pass at normal magnification using the soft copy display is accomplished by commands entered via the operator's console keyboard. The normal magnification scene is $3000 \mathrm{~km}$ square at a 1:15 million scale. For times two and four magnification, the displayed scene will be 1500 and $750 \mathrm{~km}$ square respectively. From each spacecraft pass, an area up to $8000 \mathrm{~km}$ long and $2000 \mathrm{~km}$ wide can be covered. By 1988, there will be up to 17 of these terminals operational worldwide.

\section{Shipboard Terminals}

The Navy has DMSP shipboard receiving terminals on eight carriers: the USS Midway, USS Independence, USS Kitty Hawk, USS Constellation, USS Enterprise, USS America, USS John F. Kennedy, and USS Nimitz. Imagery is provided to the afloat oceanographer to help analyze and forecast oceanographic and meteorological parameters which effect both sensor and weapon systems performance. 


\section{FUTURE PLANS}

The near future of DMSP does not foresee immediate, dramatic technological changes but, rather, a deliberate enhancement of the various subsystems based upon available technology. Primarily, these include increasing system lifetime by obtaining parts with improved reliability, that are less susceptible to radiation effects and have increased yields. Memory error correction will be incorporated into future spacecraft computer systems. Other efforts will include the incorporation of limited satellite autonomous operation, up to two months with little or no support from the ground operations centers. This will involve significant software changes as well as some spacecraft changes to achieve the stated goals.

The more distant future of DMSP looks forward to an exicting competitive program beginning in FY 88 involving a major redesign of the whole DMSP satellite and ground system. This new system which we are calling DMSP Follow-on is not projected to be operational until after the year 2000 . The competition will focus on providing the most capability within a projected life cycle cost limit based on the current 5D-3 program. All meteorological, oceanographic, and solar-geophysical requirements have been prioritized to aid potential contractors in optimizing a payload. The concept will include a 25 percent spacecraft growth capability in payload weight and power consumption, and will be dual compatible with the Shuttle as well as with an expendable launch vehicle. The ground command and control system will be compatible with future plans for the Consolidated Space Operations Center and the Transportable Mobile Ground System. In addition, several system options will be priced to determine their cost effectiveness. The options include the capability to store and forward messages to tactical terminals or small backpack terminals, the capability to measure winds with Doppler Lidar, increased levels of nuclear and laser hardness, extended autonomy up to 180 days, the capability to interrogate small data collection systems deployed on remote buoys, surface or airborne hosts, and satellite internetting for command and control flexibility and increased timely receipt of data.

\title{
SPACE AND MARITIME STRATEGY
}

\author{
Dr. E. Ann Berman \\ Deputy Assistant Secretary of the Navy \\ (Command, Control, Communications, Intelligence, \& Space) \\ Office of the Assistant Secretary of the Navy \\ Research, Engineering \& Systems \\ Washington, D.C. 20350-1000
}

\begin{abstract}
A globally deployed Navy requires and uses space systems and services. Specific mission support areas of communications, navigation, environmental data, and surveillance are discussed in terms of requirements and program developments. Navy role in pioneering space systems combined with threat assessment validates Navy position of space system acquisition and operation in the future.
\end{abstract}

\section{SPACE AND MARITIME STRATEGY}

Navy's interest in space is obvious. Space systems provide global coverage - and the Navy is globally deployed. Our

Based on a paper presented at EASCON 1986.

U.S. Government work not protected by U.S. copyright.
National Maritime Strategy has our Navy "forward deployed and in harm's way". Naval forces remain on station for extended periods of time, ready to control a crisis and project power ashore when action is required. We routinely deploy to the North Atlantic in the seas between Greenland, Iceland, and Norway. You have all read about our forces deployed in the Mediterranean off the coast of Libya and Lebanon. You may remember earlier this year, we deployed an AEGIS cruiser to the Black Sea for a brief period. We also maintain naval forces in the Indian Ocean and Persian Gulf to protect Mideast oil shipping lanes. Finally, of course, we maintain a strong pressence in the Pacific. In all these areas we are prepared to fight terrorism, to protect allied shipping from attack at sea, to exclude enemy forces from strategic ocean areas, and to project power against enemy forces ashore. Because the Navy is globally deployed, we have pioneered the use of space for communication, navigation, and environmental data collection. 